\title{
Concept And Working Of Different Types Of Fuses - Protection From Short Circuit Damages - A Bird's Eye View
}

\author{
C. Maruthi Ratna Kishore \\ Electrical and Electronics Engineering. Dept, Rise group of Institutions Ongole-523001, Andhra Pradesh, India
}

Abstract: This paper deals with the brief review of the concept and functioning of different types of fuses with a main focus on analysis of the protection available by fuses from short-circuit damages.

Keywords: Electrical fuse, Electrical Protection, Electrical faults, HRC fuses, Short-circuit currents, over currents.

\section{Introduction}

A fuse is one type of over current device that is designed to be a sacrificial element in an electrical power system. [1] Fuses are designed to open circuits when excessive currents are present due to overloads or faults and to prevent further damage to the system that might result if the fuse were not present.

The subject matter of this paper is the outcome of a bird's eye view on the concept and working of the different types of fuses. Also this paper highlights the significant role played by the fuses in providing protection from short-circuit damages. This review study may be useful to the electrical engineering graduates to put good efforts to further develop protective fuse concept, since one of the most common and potentially serious problem is a short circuit .A short can occur whenever a loose wire, dropped tool, flood of seawater, or other circumstance provides electricity with a shortcut around all or part of the regular load. Since the load normally limits the amount of current allowed to flow from the battery or other source, a short can cause a huge surge in current. This surge can quickly heat wires or other components red-hot and start a fire. A short can also bring higher voltage parts of a circuit into direct electrical contact with parts that are normally at lower voltages, so in high-voltage circuits, a short can present a dangerous electrocution hazard. Fuses are therefore selected to allow passage of normal current plus a marginal percentage and to allow excessive current only for short periods. Slow blow fuses are designed to allow higher currents for a modest amount of time longer, and such considerations are and were commonly necessary when electronics devices or systems had electronic tube tech.

The most common device in use for over current protection in high-current circuits today is the circuit breaker. Circuit breakers are specially designed switches that automatically open to stop current in the event of an over current condition. Small circuit breakers, such as those used in residential, commercial and light industrial service are thermally operated. They contain a bimetallic strip (a thin strip of two metals bonded backto-back) carrying circuit current, which bends when heated. When enough force is generated by the bimetallic strip (due to over current heating of the strip), the trip mechanism is actuated and the breaker will open. Larger circuit breakers are automatically actuated by the strength of the magnetic field produced by current-carrying conductors within the breaker, or can be triggered to trip by external devices monitoring the circuit current (those devices being called protective relays).Fuses are rated in terms of their voltage capacity as well as the current level at which they will blow. Some large industrial fuses have replaceable wire elements, to reduce the expense. The body of the fuse is an opaque, reusable cartridge, shielding the fuse wire from exposure and shielding surrounding objects from the fuse wire. Over current devices primarily protect the conductors of a circuit from over temperature damage (and the fire hazards associated with overly hot conductors), and secondarily protect specific pieces of equipment such as loads and generators (some fast-acting fuses are designed to protect electronic devices particularly susceptible to current surges). The basic purpose of the fuse is to protect and is composed of an alloy which has a low melting point. A strip of this fuse is placed in series with the circuit. The working principle is that if the current is in excess then the strip would melt and break the circuit. There are different variants of fuse boxes available with different types of circuit breaking. For instance, in the case of slow blow fuses, a small overload is carried for some period without the circuit been broken. Other fuse boxes are designed to break the circuit rapidly. The selection is based upon the kind of device and also the fluctuation level of the current. Today, semiconductor devices are being manufactured with maximum continuous current ratings up to $4 \mathrm{kA}$ and peak inverse voltages of $6 \mathrm{kV}$, [2-3]. Unfortunately, these devices still have poor overload capacities and continue to need sensitive and fast-acting protection, [4-8]. 


\section{Different types of fuses}

A brief idea of the different types of fuses and their working is given here. The main components of a standard fuse unit consist of the items: Metal fuse element, Set of contacts, Support body. The major two categories of fuses included are (1) Low Voltage Fuses, (2) High Voltage Fuses. In order to understand Low voltage fuses better, and can further classified into: Semi Enclosed or Rewireable Type, Totally enclosed or Cartridge Type.

\section{A. Rewireable Fuses}

This kind of fuse is most commonly used in the case of domestic wiring and small scale usage. Another name for this type is the Kit-Kat type fuse. The main composition is of a porcelain base which holds the wires. The fuse element is located inside a carrier that is also made out of porcelain. It is possible to remove the fuse carrier without any risk of electrical shock. Normally what happens is that when the fuse blows, it can be replaced without having to change the complete thing. The main metals or alloys used in making fuse wire include lead, tinned copper, aluminum or tin lead alloy. When there is an over surge that causes the fuse element to blow off, it can be replaced. A new fuse carrier is inserted in the base. The main advantage of this type of fuse is that it is easy to install and also replace without risking any electrical injury. Totally Enclosed or Cartridge Type In this type of fuse has a completely closed container and there are contacts (metal) on either side. The level of sub division in this case includes, D type, Link Type.

D Type Cartridge Fuses: This cannot be interchanged and comes with the following main components: fuse base and cap, adapter ring and the cartridge. The fuse base has the cap screwed to it and the cartridge is pushed into it. The circuit becomes complete when the tip of the cartridge is in contact with the conductor. In this case, the main advantage is that of reliability.

Link Type Cartridge fuses: In Link type, further a knife blade type and bolted types are available. The Knife Blade Type HRC Fuse is easily replaceable in the circuit without any load. For this purpose, special insulated fuse pullers are used. In the Bolted Type HRC Link Fuse, the conducting plates are bolted to the base of the fuse. There is also a presence of a switch through which the fuse can be removed without getting an electrical shock.

In HRC fuse or High Rupturing Capacity Fuse, the fuse wire or element can carry short circuit heavy current for a known time period. During this time if the fault is removed, then it does not blow off otherwise it blows off or melts. The enclosure of HRC fuse is either of glass or some other chemical compound. This enclosure is fully air tight to avoid the effect of atmosphere on the fuse materials. The ceramic enclosure having metal end cap at both heads, to which fusible silver wire is welded. The space within the enclosure, surrounding the fuse wire or fuse element is completely packed with a filling powder. This type of fuse is reliable and has inverse time characteristic, that means if the fault current is high then rupture time is less and if fault current is not so high then rupture time is long. The Operation of HRC fuse clearly shows that when the over rated current flows through the fuse element of High Rupturing Capacity Fuse the element is melted and vaporized. The filling powder is of such a quantity that the chemical reaction between the silver vapor and the filling powder forms a high resistance substance which very much helps in quenching the arc.

\section{B. ANL/ANE fuses}

ANL kind of fuses is mostly used in cars for the audio systems. They are available in various sizes. As opposed to others, this one has no wire terminal block. How to check the fuses? In order to check the fuse, a probe is used with readings from the terminals. The correct functioning would be when the value is $0 \mathrm{~V} \mathrm{DC}$. The fuse should be checked with the voltage being supplied. In cases, the value getting is higher than $0 \mathrm{~V} \mathrm{DC}$, this means than there is a need to remove it. In this category all fuses up to $1.5 \mathrm{kV}$ can be included. But the most typical voltage levels for low voltage fuses are $500 \mathrm{~V}, 690 \mathrm{~V}$ and $750 \mathrm{~V}$.LV HRC fuses are used for installation systems in non-residential, commercial and industrial buildings, as well as in the switchboards of power supply companies. They therefore protect essential building parts and installations. LV HRC fuse links are available in the following operational classes: $\mathrm{gG}$ (previously $\mathrm{gL}$ ) for cable and line protection, $\mathrm{aM}$ for the short-circuit protection of switching devices in motor circuits, $\mathrm{gR}$ or aR for the protection of power semiconductors, $\mathrm{gS}$ operational class combines cable and line protection with semiconductor protection.

\section{High Voltage Fuses}

All fuses used on power systems from $1.5 \mathrm{kV}$ up to $138 \mathrm{kV}$ are categorized as high voltage fuses. High voltage fuses are used to protect instrument transformers used for electricity metering, or for small power transformers where the expense of a circuit breaker is not warranted. Large power fuses use fusible elements 
made of silver, copper or tin to provide stable and predictable performance. High voltage expulsion fuses surround the fusible link with gas evolving substances, such as boric acid. When the fuse blows, heat from the arc causes the boric acid to evolve large volumes of gases. The associated high pressure (often greater than 100 atmospheres) and cooling gases rapidly quench the resulting arc. The hot gases are then explosively expelled out of the end of the fuse. Such fuses can only be used outdoors. The most frequent application is in transformer circuits, with further uses in motor circuits and capacitor banks. These types of fuses may have an impact pin to operate a switch mechanism, so that all three phases are interrupted if any one fuse blows. High-power fuse means that these fuses can interrupt several kilo amperes. Some manufacturers have tested their fuses for up to $63 \mathrm{kA}$ cut-off current.

\section{Coordination of Fuses in Series}

Where several fuses are connected in series at the various levels of a power distribution system, it is desirable to blow (clear) only the fuse (or other over current device) electrically closest to the fault. This process is called "coordination" or "discrimination" and may require the time-current characteristics of two fuses to be plotted on a common current basis. Fuses are selected so that the minor, branch, fuse disconnects its circuit well before the supplying, major, fuse starts to melt. In this way, only the faulty circuit is interrupted with minimal disturbance to other circuits fed by a common supplying fuse. Where the fuses in a system are of similar types, simple rule-of-thumb ratios between ratings of the fuse closest to the load and the next fuse towards the source can be used.

\section{E. Resettable Fuses}

So-called self-resetting fuses use a thermoplastic conductive element known as a Polymeric Positive Temperature Coefficient (or PPTC) thermistor that impedes the circuit during an over current condition (by increasing device resistance). The PPTC thermistor is self-resetting in that when current is removed, the device will cool and revert to low resistance. These devices are often used in aerospace/nuclear applications where replacement is difficult, or on a computer motherboard so that a shorted mouse or keyboard does not cause motherboard damage.

\section{F. Thermal Fuses}

These single operation devices work primarily as a temperature sensitive fuse. Due to their compact size and low cost they can be used as a primary protection device in applications where there is little thermal inertia experienced during normal operation conditions. Thermal fuses are also valuable as a cost effective added safety back-up to a primary operating control. The construction of the most common form of thermal fuses basically uses a contact spring encapsulated into a wax pellet. The pellet is formulated to melt at a predetermined maximum temperature. As the wax melts the spring stretches until it breaks the circuit in the process. Other types of thermal fuses incorporate the use of a specially formulated solder that effectively melts when exposed to given maximum temperature. These devices are non-resettable and react primarily to changes in temperature. There is some self heating effect for these devices under higher current loads which may cause the operating characteristics to change over time. Thermal fuses are also referred to as thermal links, thermal cutouts (TCO's), or one shot. A thermal fuse is often found in consumer equipment such as coffee makers, hair dryers or transformers powering small consumer electronics devices. They contain a fusible, temperaturesensitive alloy which holds a spring contact mechanism normally closed. When the surrounding temperature gets too high, the alloy melts and allows the spring contact mechanism to break the circuit. The device can be used to prevent a fire in a hair dryer for example, by cutting off the power supply to the heater elements when the air flow is interrupted (e.g., the blower motor stops or the air intake becomes accidentally blocked). Thermal fuses are a 'one shot', non-resettable device which must be replaced once they have been activated (blown).

\section{G. Surface-Mount Fuses}

Fast-Acting Chip Fuses: Our fast-acting chip fuses help protect equipment from damage caused by over current conditions when relative quick clear or open times are required in DC power applications. They reduce fuse aging, improve product reliability and resilience, and enhance high-temperature performance.

High-Current Rated Fuse: Our high-current chip fuses support surface-mount applications where space is critical and high current is required. The high reliability and strong arc suppression characteristics help make them suitable for over current protection in power supplies servers, communication equipment and more.

Slow-Blow Chip Fuses: Our slow-blow chip fuses help provide protection from damage caused by over current events on systems that experience large and frequent current surges as part of their normal operation. 
Telecom Fuses: Our telecom FT600 fuse offers low temperature-rise performance under sneak current fault events to help prevent damage to circuit traces or multilayer boards. The low profile and small footprint make them suitable for high-density and space-constrained applications.

Pulse Tolerant Chip Fuses: Pulse Tolerant chip fuses help provide over current protection on systems using DC power sources up to 63VDC. The fuse's monolithic, multilayer design provides the highest hold current in the smallest footprint, reduces diffusion-related aging, improves product reliability and resilience, and enhances high temperature performance in a wide range of circuit designs.

0603 Very Fast Acting Chip Fuses: Very fast acting at 200\% and300\% overloads. It helps provide over current protection on systems using DC power sources up to 63VDC. The fuse's monolithic, multilayer design provides the highest hold current in the smallest footprint, reduces diffusion-related aging, improves product reliability and resilience, and enhances high temperature performance in a wide range of circuit designs.

2410 Very Fast Acting Fuses: The 2410(6125) is Wire-in Air SMD Fuse which is suitable for secondary level over current protection applications. These lead-free surfaces mount devices offer reliability and have no end cap fall off risk. Straight wire element in air performs consistent fusing and cutting characteristics.

\section{H. Automotive Fuses}

Automotive fuses are used to protect the wiring and electrical equipment for vehicles. There are several different types of automotive fuses and their usage is dependent upon the specific application, voltage, and current demands of the electrical circuit. Automotive fuses can be mounted in fuse blocks, inline fuse holders, or fuse clips. Some automotive fuses are occasionally used in non-automotive electrical applications. Standards for automotive fuses are published by SAE International (formerly known as the Society of Automotive Engineers). Automotive fuses can be classified into four distinct categories: Blade fuses, Glass tube or Bosch type, Fusible links and Fuse limiters. Most automotive fuses rated at 32volts are used on circuits rated 24volts DC and below. Some vehicles use a dual 12/42 V DC electrical system that will require a fuse rated at $58 \mathrm{~V}$ DC.

\section{Fuse Device - Protection from Short Circuit Damages}

Fuse selection and device plays a predominant role in providing protection from short-circuit and other damages. It also works as a safety device in protecting human life and property. Safety by low voltage fuses and high voltage fuses in case of over currents, over loads, and short-circuits is also examined here.

\section{A. Safety in Low Voltage Fuses}

In case of low voltage fuses both in rewirible and cartridge type of fuses suitable fuse construction is made. The rewirible fuse (KIT-KAT), when blows, it can be replaced without having to complete thing. Safety fusers like cylindrical type, enclosed or cartridge type or high power rupturing capacity fuses(HRC),knife blade fuse, bolted fuse like types are providing more safety limiting the over currents and short-circuit dangerous and damages.

\section{B. Safety in High Voltage Fuses}

High power fuses can interrupt several kilo Amperes. Different types of high voltage fuses are in use to protect from overloading current and short-circuit damages. Such high voltages fuses are used to protect transformers used for electricity metering. These high power fuses are made with protective switching devices.

\section{Over Currents}

A fuse can provide protection in the event of over currents. An over current is either an overload current or a short-circuit current. The overload current is an excessive current relative to normal operating current but one which is confined to the normal conductive paths provided by the conductors and other components and loads of the distribution system. As the name implies, a short-circuit current is one which flows outside the normal conducting paths.

\section{Overloads}

A fuse can provide protection to circuits from overloads. Overloads are most often between one and six times the normal current level. Usually, they are caused by harmless temporary surge currents that occur when motors are started-up or transformers are energized. Such overload currents or transients are normal occurrences. Since they are of brief duration, any temperature rise is trivial and has no harmful effect on the circuit components. (It is important that protective devices do not react to them.) Continuous overloads can result from defective motors (such as worn motor bearings), overloaded equipment, or too many loads on one 
circuit. Such sustained overloads are destructive and must be cut-off by protective devices before they damage the distribution system or system loads. However, since they are of relatively low magnitude compared to shortcircuit currents, removal of the overload current within a few seconds will generally prevent equipment damage. A sustained overload current results in overheating of conductors arid other components and will cause deterioration of insulation which may eventually result in severe damage and short-circuits if not interrupted.

\section{E. $\quad$ Short - Circuits}

A fuse can offer protection to circuits from short-circuits. Whereas overload currents occur at rather modest levels, the short-circuit or fault current can be many hundreds of times larger than the normal operating current. A high level fault may be 50,000 Amps (or larger). If not cut off within a matter of a few thousands of a second, damage and destruction can become rampant-there can be severe insulation damage, melting of conductors, vaporization of metal, ionization of gases, arcing, and fires. Simultaneously, high level short-circuit currents can develop huge magnetic-field stresses. The magnetic forces between bus bars and other conductors can be many hundreds of pounds per lineal foot; even heavy bracing may not be adequate to keep them from being warped or distorted beyond repair.

\section{F. The concept of Controllable Fusing}

With the aim to improve the fuse features, a new concept of controllable fusing has been patented. The controllable fusing means the possibility of fuse to operate at certain time moments when an external command is activated. The key element of the controllable fusing is an electrode which is placed on the fuse link element. The electrode is made from graphite and is pressed on the copper strip of the fuse element. The electrode terminal is made from brass in order to allow a good contact with the supply conductor. With the aim to supply this electrode, a detachable contact, or a plug device is used in the case of more parallel fuse link elements, [910]. Thus, when an over current occurs within an electric circuit where a high breaking capacity fuse is mounted to protect a device against over currents, especially short-circuits, some transducers which are sensitive at current value, di/dt, temperature, etc., will provide a command signal for a power switch. This switch will supply with the necessary power the electrode, which finally will interrupt the fuse element, through an auxiliary electric arc. Therefore, the fuse will turn off the main electric circuit.

\section{Conclusion}

In the light of the above review study a clear and brief summary and conclusion is drawn here. The concept of different types of fuses concentrated on 'safety and protection' to minimize the damage from shortcircuit and over currents. A fuse is a small, thin conductor designed to melt and separate into two pieces for the purpose of breaking a circuit in the event of excessive current. A circuit breaker is a specially designed switch that automatically opens to interrupt circuit current in the event of an over current condition. They can be "tripped" (opened) thermally, by magnetic fields, or by external devices called "protective relays," depending on the design of breaker, its size, and the application. Fuses are primarily rated in terms of maximum current, but are also rated in terms of how much voltage drop they will safely withstand after interrupting a circuit. Fuses can be designed to blow fast, slow, or anywhere in between for the same maximum level of current. The theoretical aspects related to the new type of high breaking capacity fuse based on the controllable fusing effect and all the experimental tests outline that there is the possibility to extend the current protection range both to overloads and short-circuits. Thus, the traditional passive over current protection becomes an active one enhanced with new features such as, Controllable fusing level, Controllable current-limiting effect, adjusted time-current characteristics, Protection possibilities from overload to short-circuits, Protection to direct current sense and power line sense at AC applications, Protection to di/dt. Extending the new concept of controllable fusing for the specific applications enables the user of high breaking capacity fuses to choose the right ratings, to evaluate critical load cycles and to identify potential overload capacities for a dynamic grid loading. It was shown that the described new fuse has a high potential for a variety of different applications such as, development support, identifying user risks. Though the existing fuses are providing possible protection from short-circuit damages, still incidence of damages, fire-hazards are taking place often in many places, both in household and industrial applications. So still more efforts are necessary to develop newly designed and more safety and protective fuses which can limit the danger from short-circuit to the maximum extent.

\section{References}

[1] John Wiles, "Focusing On Fuses", www.nmsu.edu_tdi_pdf-resources_cc67, Home Power Magzine\#67 • October / November $1998, p p .100$.

[2] Al-Hajri M T (2008). Power losses due to pipeline cathodic protection (CP) 6-pulse rectifier, Conference Record of the IEEE International Symposium on Electrical Insulation, pp.514-517.

[3] Robless D B, and King R J (1992). A 1 kW unity-power - factor rectifier with isolation and fault protection, Seventh Annual Applied Power Electronics Conference and Exposition,pp. 541-548.

[4] Salomonsson D, Soder L et al. (2009). Protection of low-voltage DC microgrids, IEEE Transactions on Power Delivery, vol.24 
,3,pp. 1045-1053.

[5] Eui - Cheol N, In-Dong, K et al. (2001). An improved output short - circuit protection for multilevel PWM rectifier, The 27th Annual Conference of the IEEE Industrial Electronics Society, vol 2, pp. 909-914.

[6] Galloway J H, and Buddingh P (2010). Protection of large power conversion equipment, 57th Annual Petroleum and Chemical Industry Conference, Industry Applications Society, pp. 1-7.

[7] Livint G,Chiriac $\mathrm{G}$ et al. (2005).Aspects regarding the hybrid electric vehicles architecture and the management strategy for the energy flow, The 4th Management of Technological Changes Conference (Chania), pp. 275-280.

[8] Albu M, Lucache D et al. (2005). Flexible power MOSFET topology for the automotive DC - DC converters study, Buletinul Institutului Politehnic Iaşi, vol LI(LV), pp. 217-224.

[9] Chiriac G (2012). Thermal analysis of fuses with variable cross-section fuselinks, Electric Power Systems Research, Vol 92, pp. $73-80$.

[10] Cantemir L, Nituca C et al . (2011). Unconventional current collection from a contact line for electric traction vehicles , International Conference on Pantograph Catenary Interaction Framework for Intelligent Control, Amiens, France.

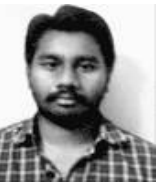

C.Maruthi ratna kishore is born in Andhra Pradesh, INDIA. He is with Electrical \& Electronics Engineering Department, Rise group of engineering institutions, affiliated to Jawaharlal Nehru Technical University Kakinada (JNTUK), Ongole-523001, Andhra Pradesh INDIA. He has contributed several research papers in the field of Electrical engineering to various International journals. 